\title{
Space astrometry with the Joint Milliarcsecond Astrometry Pathfinder
}

\author{
Gregory S. Hennessy ${ }^{1}$ and Ralph Gaume ${ }^{2}$ \\ ${ }^{1}$ US Naval Observatory, 3450 Massachusetts Ave NW \\ Washington, DC 20392, USA \\ email: gsh@usno.navy.mil \\ ${ }^{2}$ US Naval Observatory, 3450 Massachusetts Ave NW \\ Washington, DC 20392, USA \\ email: rgaume@usno.navy.mil
}

\begin{abstract}
The Joint Milliarcsecond Astrometry Pathfinder Survey (JMAPS) is a small, spacebased, all sky, visible wavelength, astrometric and photometric survey mission for $0^{\text {th }}$ through $14^{\text {th }}$ I-band magnitude stars with a planned 2013 launch. The primary objective of the JMAPS mission is the generation of an astrometric star catalog with 1 milliarcsecond (mas) positional accuracy or better and photometry to the $1 \%$ accuracy level or better at $1^{\text {st }}$ to $12^{\text {th }}$ mag. A 1-mas all-sky survey will have a significant impact on our current understanding of galactic and stellar astrophysics. JMAPS will improve our understanding of the origins of nearby young stars, provide insight into the dynamics of star formation regions and associations, investigate the dynamics and membership of nearby open clusters.
\end{abstract}

Keywords. astrometry, space vehicles, catalogs, surveys

\section{Introduction}

JMAPS is a small, single aperture spacecraft funded by the Department of the Navy for launch in 2013. The principal objective of the JMAPS mission is to produce an all-sky, visible wavelength, astrometric and photometric catalog (Zacharias \& Dorland 2006). The final JMAPS catalog will be delivered in 2016. Astrometric positions will be reported in ICRS coordinates and tied to the ICRF though direct observations of the visible wavelength counterparts of radio wavelength ICRF sources. An artist's conception of JMAPS, both telescope and bus, is depicted in Figure 1.

\section{SPACECRAFT \& INSTRUMENT OVERVIEW}

\subsection{Spacecraft}

As shown in Figure 1, JMAPS is a single spacecraft consisting of a customized spacecraft bus (bottom) with solar panels and payload deck (top). The solar panels fold against the bus in a stowed and locked position during launch and are deployed on-orbit. In addition to housing the power subsystem, the spacecraft bus contains communications, thermal control, avionics, reaction wheel, and inertial measurement unit subsystems. The Attitude Determination and Control System (ADCS) is split between the bus and instrument deck. While the spacecraft is slewing, the star tracker located on the instrument deck determines spacecraft attitude to approximately 1 arcsecond. During standard observations the ADCS system holds spacecraft pointing stability to a 50 mas specification. This is accomplished by using the primary instrument to generate boresight pointing quaternions at a $5 \mathrm{~Hz}$ rate as derived from observations of reference stars on the focal plane. The 


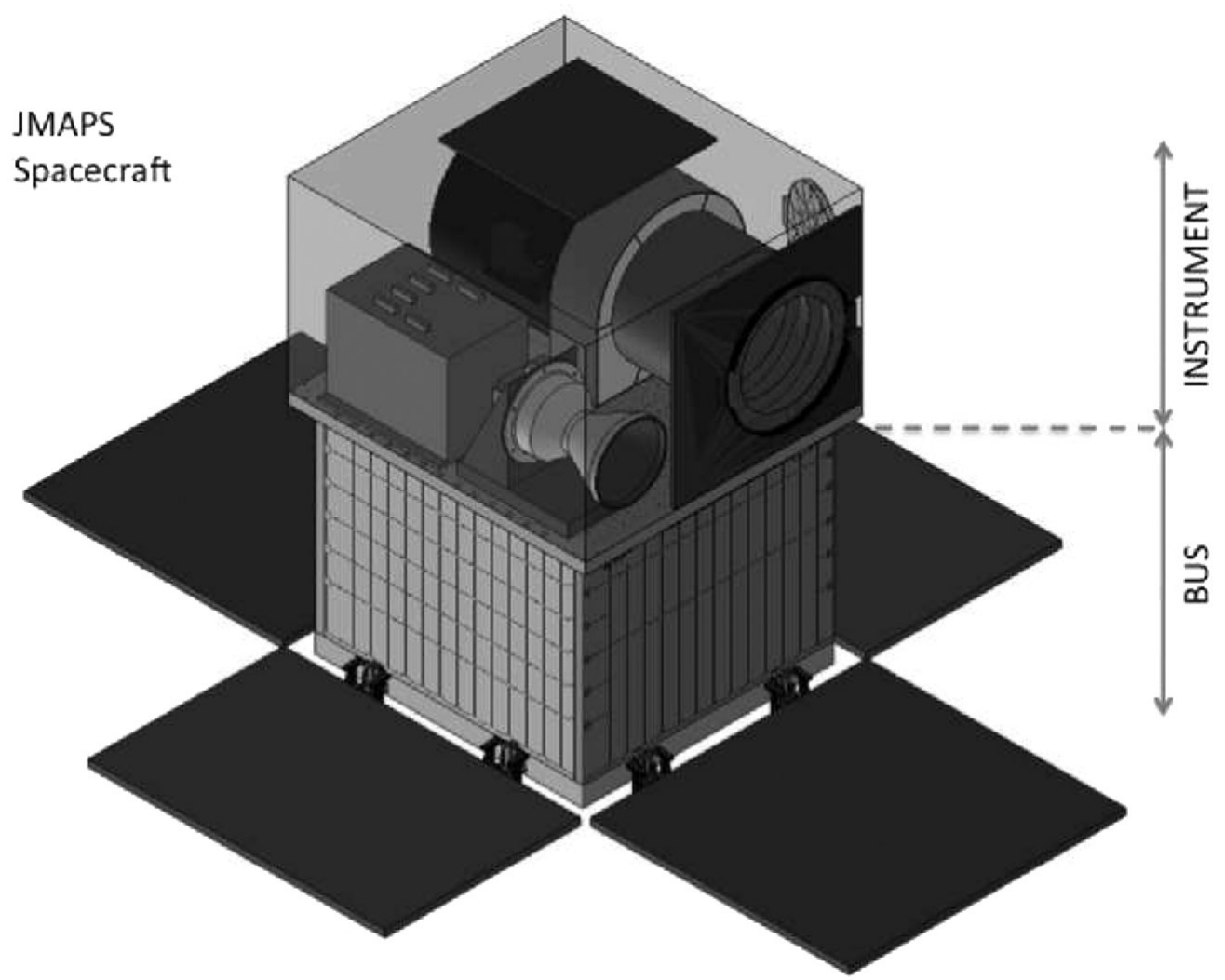

Figure 1. The JMAPS instrument and spacecraft bus.

\begin{tabular}{c|c}
\hline JMAPS & Hipparcos \\
Single aperture & two apertures \\
Step-Stare & Scanning \\
Complete to 14th mag & Complete to 7th mag \\
20 years of proper motion for Hipparcos/Tycho stars & 3 years of proper motions \\
Three years proper motions for others & \\
Low earth, approximately circular & Highly eccentric orbit \\
\hline
\end{tabular}

Table 1. Comparison of JMAPS and Hipparcos

total mass of the spacecraft, including contingency, is about $180 \mathrm{~kg}$ and the spacecraft occupies a volume of $96.5 \mathrm{~cm}(\mathrm{~h}) \times 71 \mathrm{~cm} \times 61 \mathrm{~cm}$.

Many of the stars JMAPS will observe are stars observed by Hipparcos. One of the major scientific goals of JMAPS is the production of a catalog that has new milliarcsecond precision. The expected mean epoch of 2014.5 for JMAPS combined with the Hipparcos milliarcsecond precisions at 1991.25 will provide proper motions for 118,000 stars at accuracies of about 0.05 mas/year. We provide a comparison between the JMAPS and Hipparcos spacecraft in Table 1. A primary goal for JMAPS is to produce a star catalog in support of next generation star trackers. Multiple non destructive reads of the focal plane, and rapid windowing around the brightest stars will be used to obtain the 15 magnitudes of dynamic range. JMAPS will observe approximately the same dynamic range as Gaia, but will be observing brighter stars. 


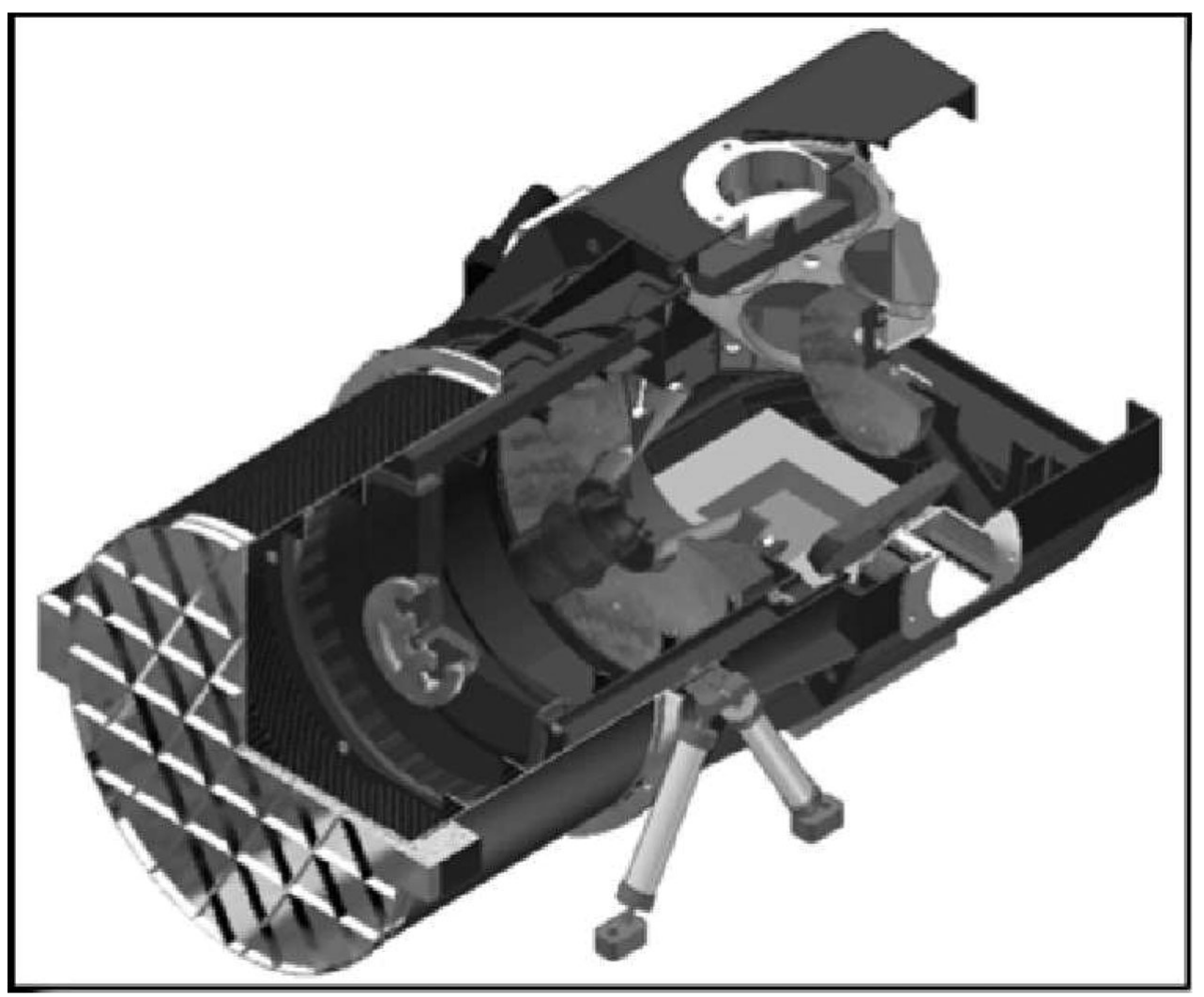

Figure 2. A view of the JMAPS optical system.

\subsection{Instrument}

The Optical Telescope Assembly (OTA) is the largest structure on the payload deck, consisting of a single aperture, $19 \mathrm{~cm}$ diameter, f/20 telescope (Dorland and Dudik 2009). A cut-away view of the telescope is visible in Figure 2. A ray trace diagram is shown in Figure 3.

The nominal point spread function (PSF) is 0.87 arcseconds full width half maximum (FWHM). The JMAPS field of view is $1.24^{\circ} \times 1.24^{\circ}$. The OTA supports a JMAPS astrometric bandpass of 700-900 nm; spectroscopic observations will be conduced within a wider 450-900nm band. During normal observations, a sun shield behind the OTA (not shown in Figure 1) will protect the OTA from direct exposure to solar heating.

The Focal Plane Assembly (FPA) is located under the OTA (the shaded square in Figure 2). The JMAPS FPA consists of a $2 \times 2$ mosaic detectors as shown in Figure 4 . The detector is a 10 micron pitch, $4192 \times 4192$ pixel CMOS-CCD hybrid. The nominal pixel subtense is 0.55 arcseconds, providing a sampling of approximately 1.6 pixels per PSF FWHM. USNO has sky-tested the first generation detector (Dorland et al. 2007). Second generation detectors have been built and are currently undergoing testing at USNO. It is anticipated that JMAPS flight devices will result from the third generation of the H4RG-10 detector.

Major components of the payload deck (also shown in Fig. 3) are the Instrument Electronics Box (IEB)-which houses the primary on-board electronics for both the instrument and bus-the star tracker and FPA radiators. The radiators will be used to maintain the JMAPS FPA at a temperature of 193K. 


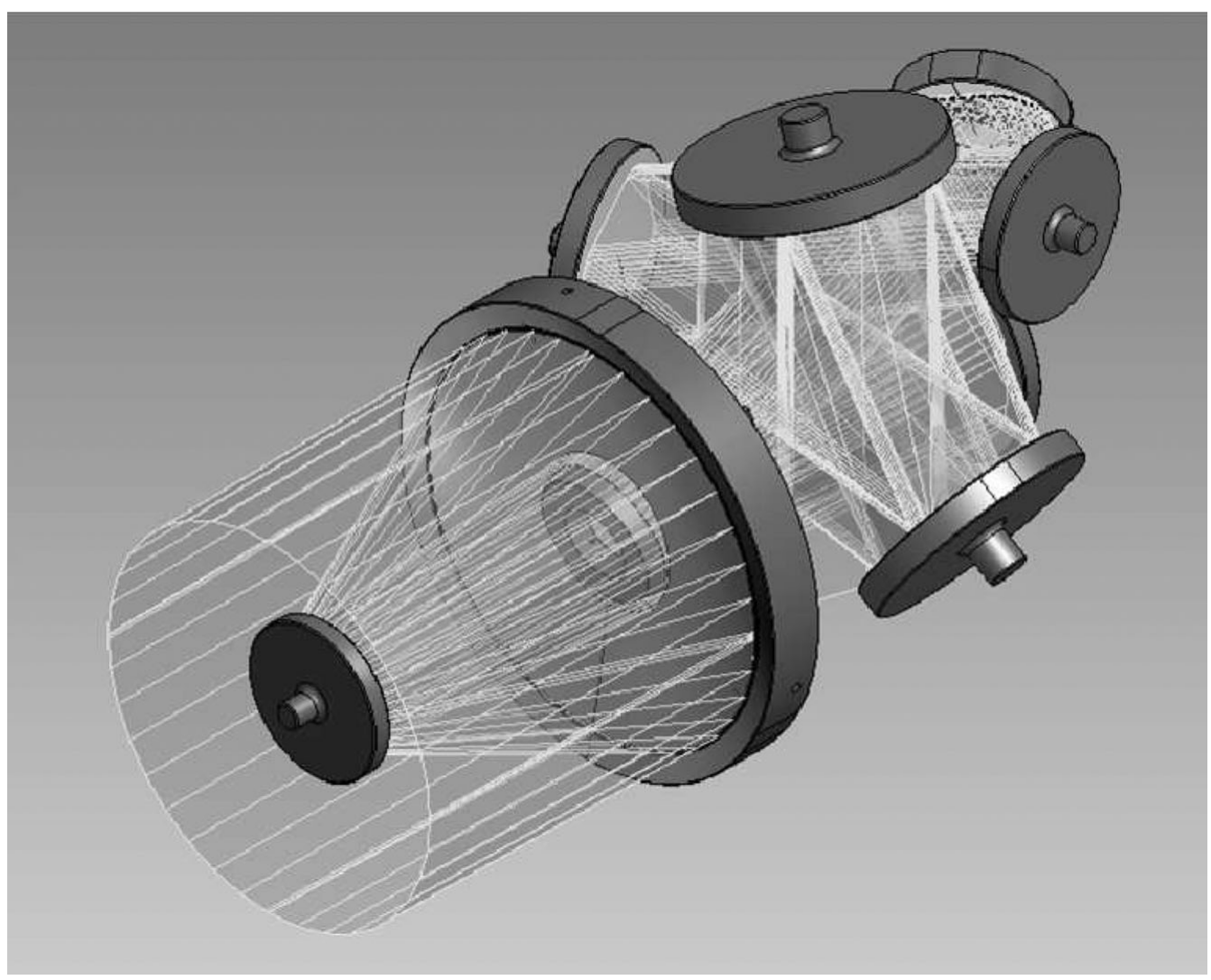

Figure 3. A ray tracing of the JMAPS optical system.

\subsection{JMAPS Concept of Operations}

JMAPS will be launched into a $900 \mathrm{~km}$ sun-synchronos terminator orbit and operate in a step-stare mode, typically sweeping out swaths of the sky at approximately a $90^{\circ}$ angle to the Earth-Sun line (regions of maximum parallax signal). Windowed FPA integration times of 1, 4.5 and 20 seconds will be used for the majority of stars. Shorter integration times (between $10 \mathrm{msec}$ and $1 \mathrm{sec}$ ) will be used for the brightest stars. In order to link the JMAPS reference frame to the ICRF, the optical counterparts of radio wavelength ICRF quasars will be directly observed by JMAPS by employing integration times of up to 500 seconds.

\section{Measurements}

\subsection{Single field astrometric}

The majority of observations will be taken in quadrature with the sun, in order to maximize the parallax factor. The angle between the boresite and the sun will be allowed to vary by up to 20 degrees from quadrature. After an exposure is taken the boresite will move half a field for the subsequent exposure. Due to the limited on-board data storage capacity, full frames will not be downlinked from the spacecraft. Instead an onboard processor will identify stars, remove cosmic rays and then download a $10 \times 10$ pixel window centered on each star. These windows will be processed on the ground to determine the centroid and instrumental magnitude of each star. 


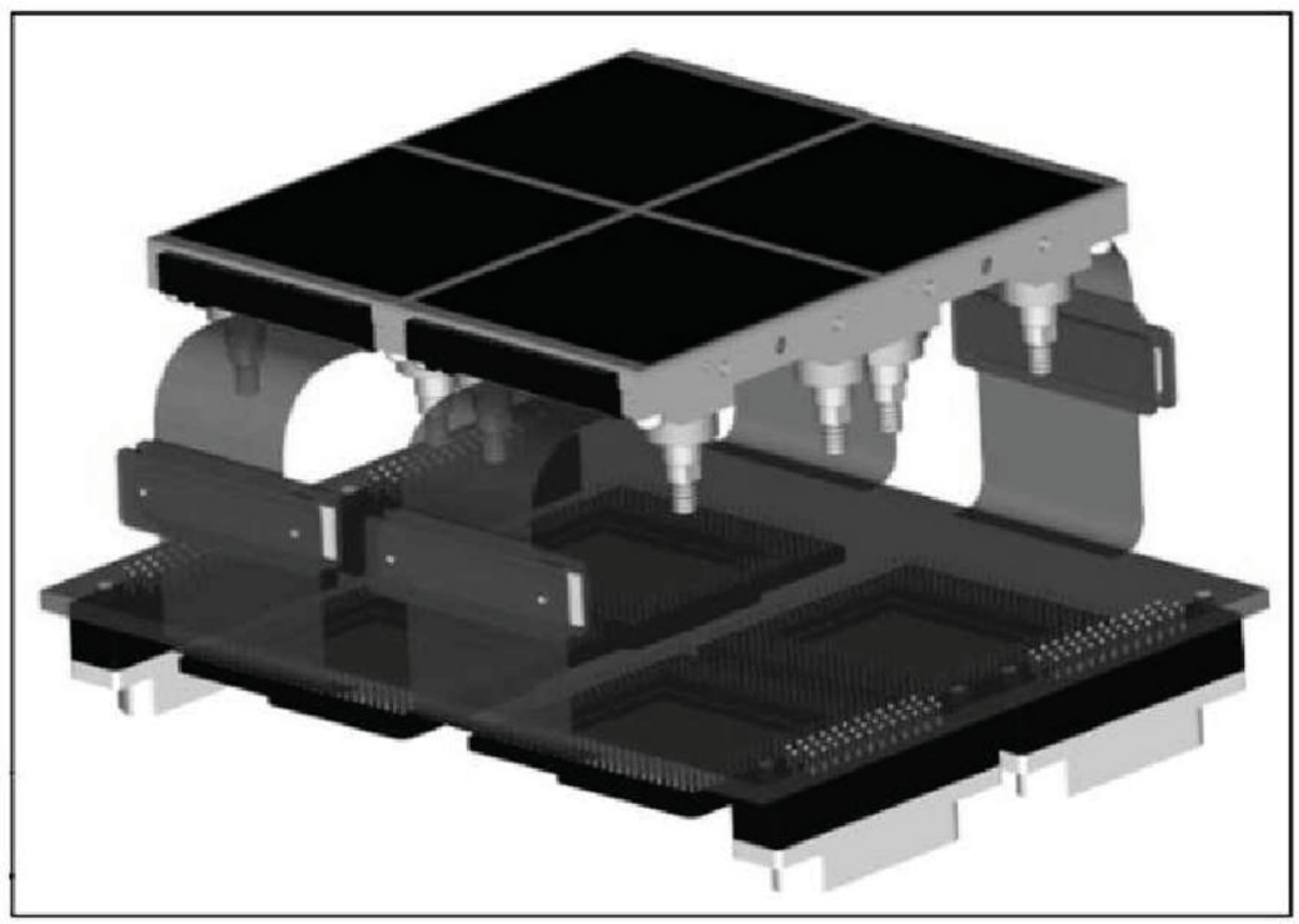

Figure 4. JMAPS FPA $4 \mathrm{~K} 2 \times 2$ Mosaic Concept

\subsection{Global Reduction}

Global block adjustment algorithms will be used to provide a rigid determination of place centers and rotations for each of the exposures. The use of approximately 100 optically bright quasars that are also part of the International Celestial Reference Frame will be used to place the JMAPS results on an inertial frame. A catalog of 400,000-600,000 "grid stars" will be used to calculate the per frame plate constants. The use of a global reduction is expected to reduce, but not eliminate, zonal errors.

\section{JMAPS SCIENCE POSSIBILITIES}

The predicted JMAPS single measurement precision (SMP) is shown in Figure 5. The sawtooth nature of the SMP plot is attributed to the various integration times utilized for stars of different magnitudes (see Figure 5). The objective of achieving 1 mas stellar astrometric accuracies is achieved through a global reduction of multiple (approximately 72) observations obtained throughout the 2-3 year mission lifetime. A 1mas all-sky survey will have a significant impact on our current understanding of galactic and stellar astrophysics. JMAPS will improve our understanding of the origins of nearby young stars, provide insight into the dynamics of star formation regions and associations, investigate the dynamics and membership of nearby open clusters, discover the smallest brown dwarfs at distances up to 5 pc after a 2-year mission and Jupiter-like planets out to 3 pc after 4 years. JMAPS will provide critical milliarcsecond-level parallaxes of tens of millions of stars in the difficult $8-14^{t h}$ magnitude range, which when combined with stellar spectroscopy and relative radii determined from exoplanet transit surveys, allows a determination of stellar radii and exoplanet densities. 


\section{Final Single Measurement Precision}

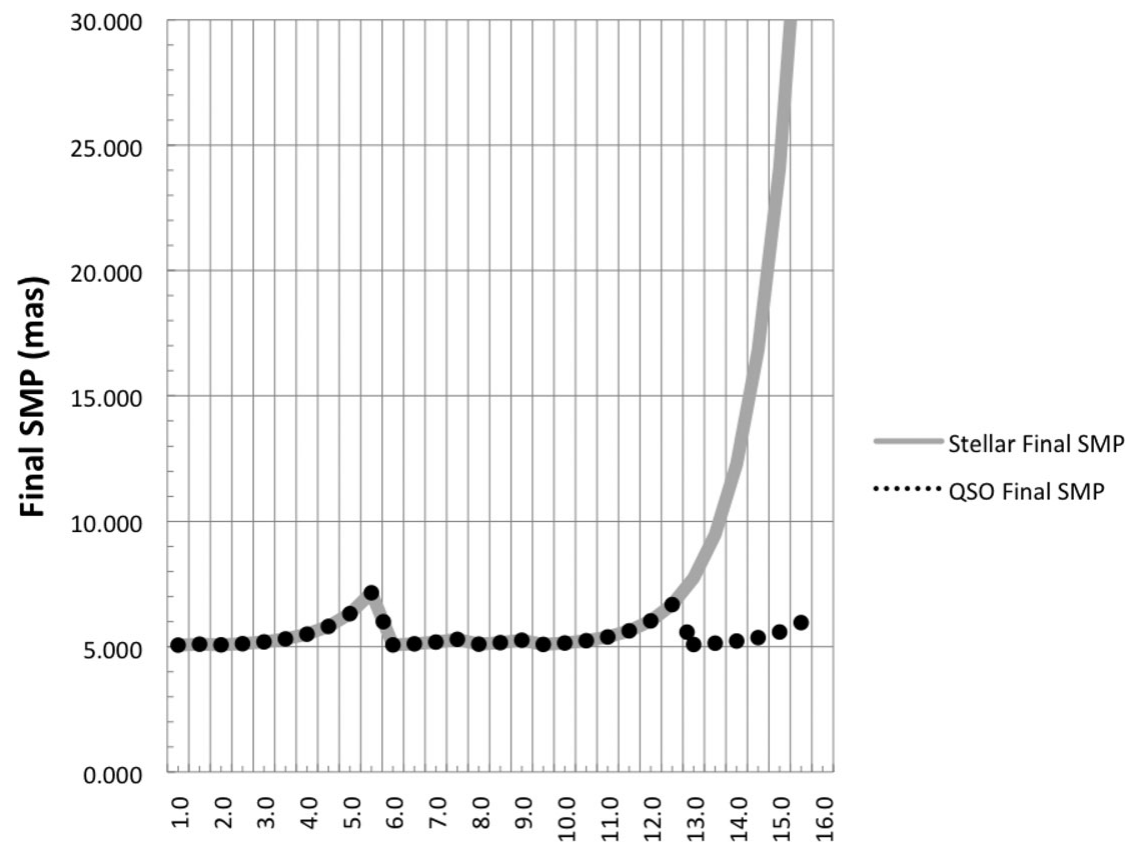

I-band magnitude

Figure 5. Estimated JMAPS single measurement precision predictions as a function of stellar I-band magnitude

\section{References}

Dorland, B. N. \& Dudik, R. P. 2009, arXiv:0907.5248

Dorland, B. N., Hennessy, G. S., Zacharias, N., Rollins, C., Huber, D., \& Kessel, R. 2007, Proc. SPIE, 6690

Zacharias, N. \& Dorland, B. 2006, PASP, 118, 1419 